\title{
Rire grand siècle et rire fin de siècle : Catulle Mendès lecteur de Charles Perrault
}

Bertrand Vibert

\section{(2) OpenEdition}

\section{Journals}

\section{Édition électronique}

URL : http://journals.openedition.org/recherchestravaux/275

DOI : 10.4000/recherchestravaux.275

ISSN : 1969-6434

Éditeur

UGA Éditions/Université Grenoble Alpes

\section{Édition imprimée}

Date de publication : 30 mai 2005

Pagination : 145-159

ISBN : 0151-1874

ISSN : 0151-1874

Référence électronique

Bertrand Vibert, "Rire grand siècle et rire fin de siècle : Catulle Mendès lecteur de Charles Perrault », Recherches \& Travaux [En ligne], 67 | 2005, mis en ligne le 18 décembre 2013, consulté le 08 septembre 2020. URL : http://journals.openedition.org/recherchestravaux/275 ; DOI : https://doi.org/10.4000/ recherchestravaux.275 


\section{Rire grand siècle et rire fin de siècle: Catulle Mendès lecteur de Charles Perrault}

La tradition a tendance à estomper le caractère plaisant, voire franchement comique des contes merveilleux littéraires les plus justement célèbres de notre littérature ${ }^{\mathrm{I}}$. On aura reconnu les Histoires ou Contes du temps passé de Charles Perrault - dont on a par ailleurs surtout retenu le sous-titre: Contes de ma mère l'Oye. C'est d'abord la rançon de leur gloire: connus de tous, très souvent racontés et adaptés, les contes finissent par vivre au fil des générations comme ils avaient vécu avant d'être consignés par écrit - indépendamment de la lettre et de la manière de raconter de celui qui les a recueillis et qui est devenu leur auteur pour la postérité ${ }^{2}$. Mais c'est aussi qu'il semble exister une sorte de contradiction a priori entre l'adhésion naïve au merveilleux des contes - destinés aux enfants sous prétexte qu'ils auraient été à l'origine des contes de nourrice -, et la distance à l'égard du merveilleux qu'entraîne nécessairement un regard ironique ou comique sur les événements de la fable. Or cette distance est manifeste dans les contes merveilleux au second degré d'une autre fin de siècle (le XIXe), où certes on ne s'étonne guère de la trouver, tant l'esprit de la décadence semble par définition à l'extrême opposé de cette fraîcheur naïve que l'on se plaît à associer aux contes. C'est aussi pourquoi les contes fin de siècle 3 éprouvent le besoin de se situer par rapport à cet âge d'or - évidemment mythique - d'un genre en particulier représenté par Perrault et Madame d'Aulnoy. Or, à y regarder de près, les choses pourraient être moins simples, si l'on veut bien admettre qu'il n'y a pas beaucoup plus de naïveté chez l'auteur de «La Belle au bois dormant» que chez celui de «La Belle au bois rêvant»,

I. Bien entendu, cet aspect n'a pas échappé à la tradition critique, par définition savante, mais il n'est pas sûr que cela ait beaucoup influé sur la réception des contes elle-même.

2. Je n'entre pas ici dans la querelle ou le procès d'attribution en paternité des Contes de Perrault entre le père et le fils, sous le nom de Pierre Darmancour. C'est seulement la signature Perrault - sa manière et son style - qui sera évoquée ici.

3. L'expression renvoie ici aux contes de la fin du XIX ${ }^{e}$ siècle. 
entendez Catulle Mendès. Ce n'est pas qu'il s'agisse de lire chez Perrault les prémices de ces Perversions du merveilleux qu'a fort bien analysées Jean de Palacio (Séguier, 1993). Mais au-delà de différences globales fort évidentes - qui tiennent à la nature de projets et d'esthétiques chez des écrivains appartenant à des champs littéraires et sociaux historiquement distincts et lointains -, on voudrait néanmoins montrer que de Perrault à Mendès peuvent se lire des convergences qui tiennent à une certaine affinité pour le comique, faite d'esprit enjoué et de connivence avec le lecteur, le tout sur fond de conscience métatextuelle aiguë4. Chez l'un comme chez l'autre, le plaisir de conter des histoires merveilleuses n'implique aucune naïveté. Surtout, le plaisir qu'il procure à l'auteur - et on l'espère aussi au lecteur - ne contredit en rien celui de rire, voire de rire des contes eux-mêmes. Si, touchant Mendès, il est clairement établi que ses contes s'adressent à un public d'adultes et de lettrés, il n'y aurait donc pas lieu de s'étonner que ceux de Perrault, dans leur forme originelle, risquent fort aujourd'hui de toucher le même public's, même si ce ne fut pas le premier visé par l'auteur. Chez tous les deux, la portée du comique dépasse en effet bien souvent la vision du monde et même de l'univers des contes que peut concevoir un enfant. Et quoique cette fantaisie comique se donne aisément carrière dans la satire, elle est souvent emportée - comme on le verra ensuite - par une manière purement ludique de raconter. C'est sur ces bases que, pour finir, on lira chez Mendès cette espèce singulière d'hommage qu'est la parodie.

\section{La satire, ou la part de réel des contes}

On sait à quel point Perrault ne dédaigne pas le trait ironique et volontiers satirique. C'est dire que le conte merveilleux touche ici d'autant plus la réalité sociale que, par sa définition même, il est censé s'en abstraire. Ce faisant, l'auteur s'amuse tout en s'offrant une sorte de victoire imaginaire; et le cas

4. Je rejoins ici la thèse de J.-P. Sermain dans Métafictions (I670-I730), La Réflexivité dans la littérature d'imagination, Paris, Champion, "Les dix-huitièmes siècles», 2002, p. 358: "Le conte de fées correspond exactement à l'idée de "métafiction" puisqu'il prend pour objet une fiction et se constitue comme réflexion à cet égard.» Perrault, sans parler de Mendès! serait à cet égard tout à fait exemplaire.

5. Sans doute une telle affirmation fait-elle un peu trop bon marché d'un Perrault moraliste et éducateur - et de ses propres enfants pour commencer. À cet égard, il convient néanmoins de méditer la remarque de $\mathrm{M}$. Soriano: "Ce que [...] nous pouvons affirmer, c'est que replacés à leur époque, ces contes sont bien un livre pour enfants. Un livre pour les enfants de l'époque." (Les Contes de Perrault. Culture savante et traditions populaires, Paris, Gallimard [Bibliothèque des idées], I973, p. 343. Mais le constat n'appelle aucune exclusive, comme le prouvent assez le choix de dédicataires adultes, le succès de salon immédiat des contes et plus sûrement encore leur analyse interne. 
échéant, le lecteur y gagne aussi en plaisir de connivence. On a ainsi souvent remarqué que Perrault prenait en particulier les femmes pour cibles privilégiées. Par là il ne fait guère que s'inscrire dans une longue tradition littéraire qui remonte au moins aux fabliaux et qui en fait un topos sans risques. Les exemples abondent en effet dans les contes, qui vont de l'ironie souriante sur la coquetterie des femmes dans "Cendrillon" par exemple - lorsque toutes les dames du bal souhaitent imiter la toilette de Cendrillon pour la fête du lendemain $^{6}$ - à des formes qui semblent quelquefois nettement plus appuyées voire méchantes. Ainsi de la seconde moralité de «La Barbe bleue», qui argue de l'ancienneté du conte pour décocher in fine une flèche venimeuse à la gent féminine tout entière. Certes, la légèreté et la curiosité de la jeune épouse étaient peu épargnées par le conteur, mais au moins sa faiblesse trop humaine l'était-elle par la fin du conte au regard de la cruauté de son barbare époux. Or, la seconde moralité à son tour renverse purement et simplement le sens de la fable et, non sans désinvolture, lui dénie toute validité:

\section{Il n'est plus d'Époux si terrible, Ni qui demande l'impossible, Fût-il mécontent et jaloux.}

Près de sa femme on le voit filer doux;

Et de quelque couleur que sa barbe puisse être, On a peine à juger qui des deux est le maître. (Éd. citée p. 266)

Paradoxalement, de façon assez retorse, on voit ici Perrault déléguer aux vérités supposées du temps présent le soin de démentir celles du passé - entendez celles des contes -, tout en feignant d'ignorer que ces vérités sont ellesmêmes largement puisées dans un héritage culturel très ancien. Mais au fond, la connivence repose sans doute moins sur le bien-fondé de l'affirmation que sur la communauté d'esprit et de culture qu'elle établit. C'est pourquoi il n'est nullement nécessaire d'être tant soit peu misogyne pour la goûter. En revanche, la misogynie ne saurait y suffire sans la détente d'esprit qu'elle implique. Néanmoins, on perçoit aussi chez Perrault des accents satiriques plus personnels comme dans "Le Petit Poucet», lorsque le bûcheron s'impatiente des reproches que lui fait sa femme pour avoir perdu leurs enfants dans la forêt:

Ce n'est pas que le Bûcheron ne fût peut-être encore plus fâché que sa femme, mais c'est qu'elle lui rompait la tête, et qu'il était de l'humeur de beaucoup d'autres gens, qui aiment fort les femmes qui disent bien, mais qui trouvent très importunes celles qui ont toujours bien dit. (p. 292)

6. Éd. de M. Soriano, Paris, Garnier-Flammarion, I99I, p. 280: «Toutes les Dames étaient attentives à considérer sa coiffure et ses habits, pour en avoir dès le lendemain de semblables, pourvu qu'il se trouvât des étoffes assez belles, et des ouvriers assez habiles. " Toutes les citations renvoient à cette édition, dont elles conservent l'orthographe. 
La généralisation progressive (il était de l'humeur de beaucoup d'autres gens»), la concession savamment ménagée ("qui aiment fort les femmes qui disent bien»), et l'implacable antithèse qui suit - fondée principalement sur l'opposition des temps, et suggérant perfidement que précisément "celles qui ont toujours bien dit» ne seraient jamais celles «qui disent bien" - relèvent d'une satire directe, non voilée, ni récupérable au nom de valeurs culturelles nécessairement partagées. Même s'il ne manque pas d'esprit, le conte semble rejoindre ici le sérieux de la vie réelle. On peut supposer que le caractère gnomique de la formule n'en fait pas moins dépendre son approbation du jugement de «beaucoup [de] gens» qui sont explicitement pris à témoins. Lecteurs, en sommes-nous? Acceptons-nous d'en être, au nom du brillant de la formule? À coup sûr oui, mais il n'empêche que le conte de fées y fait la preuve - si elle était à faire - qu'il ne saurait jamais perdre de vue tout à fait le réel ni le sérieux, même s'il en fait un objet de raillerie.

On retrouvera chez Catulle Mendès, sinon cette forme d'engagement personnel qu'on trouve chez Perrault, du moins l'appel à la complicité du lecteur dans la satire. La coquetterie y est souvent évoquée, comme dans "Le Miroir", conte dans lequel une reine, "laide au point que les pires monstres auraient paru charmants auprès d'elle $^{7}$ ", a fait briser et interdire tous les miroirs dans son royaume:

La désolation allait donc au-delà de ce qu'on peut imaginer, surtout chez les personnes coquettes qui n'étaient pas plus rares dans ce pays que dans les autres [...]. (p. 78)

La satire est bien présente, mais bénigne, et elle est s'accompagne d'un jeu ironique. "Dans les autres", qu'est-ce à dire? Les autres royaumes du passé - et donc des fables - sans doute, mais tout aussi bien «le nôtre» dont le conte nous offre, au moins à cet égard, un miroir très fidèle. Pour le coup, on ne passe pas vraiment de l'autre côté du miroir en entrant dans le royaume de la féerie, car - ainsi que le conte nous le suggère - il existe dans tous les royaumes des traits constants, parmi lesquels il faut compter la coquetterie des femmes.

Si la satire semble plus légère chez Mendès que chez Perrault, elle n'en est pas moins quelquefois plus sombre sur le fond, lorsqu' elle prend pour objet les étranges passions de l'âme humaine: moins dirigée sur des cibles particulières que celle de son devancier - les femmes, ou encore les mœurs du peuple et ses superstitions -, elle se développe néanmoins tous azimuts, dégageant une vision du monde qui ne va pas non plus sans méchanceté, dès lors que celle des personnages se trouve relayée par le cynisme du conteur. Ainsi, dans « La

7. Les Oiseaux bleus, éd. J. de Palacio, Paris, Séguier (Bibliothèque décadente), 1993, p. 77. Toutes les citations de Catulle Mendès renvoient à ce recueil. 
Bonne Récompense», une jeune princesse se trouve inconsolable, et le roi son père fort affligé lui-même:

Il n'avait de goût à rien, ne s'intéressait plus aux affaires de l'État, bâillait aux flatteries de ses courtisans; c'en était au point qu'il assista un jour, sans la moindre satisfaction, à la pendaison de deux ministres, bien que les spectacles de cette espèce eussent toujours eu le privilège de le mettre de belle humeur. (p. 136)

Il semble que le prisme de Voltaire s'interpose ici entre Perrault et Mendès. On peut y lire une forme d'excès dans la noirceur qui convient aussi à la décadence. Le ton de l'ironie légère signe bien pourtant la manière de Mendès: ironie, ou bien humour, s'il s'agit, non pas de "présenter comme sérieuse une chose que l'on ne pense pas sérieusement", mais bien plutôt de "plaisante[r] sur un sujet sérieux ${ }^{8}$ »? Le masque du conteur est en fait d'une opacité qui ne laisse rien transparaître. Car on n'est pas très sûr que le conteur faussement naïf ne vise pas contradictoirement deux cibles distinctes, les ministres qu'il nous offre en pâture quand même, et le roi monstrueusement frivole ordonnateur de leur supplice. Si ce n'est là qu'un détail presque oiseux par rapport à l'économie générale du conte, il suffit pourtant à montrer la futilité de ce qui en fait l'objet principal: le chagrin de la princesse. De ce point de vue, la satire ne manque pas de servir l'esthétique générale du conte de fées: elle établit le merveilleux après en avoir récusé les fondements, lui interdisant dès lors toute naïveté, toute mièvrerie - et il faut ajouter sans ambages, en songeant à ce qui régit aujourd'hui la plus grande production du merveilleux destiné aux enfants (dont les adultes eux-mêmes): le kitsch 9. Fiction, certes, mais sans nécessiter l'alibi du mensonge, le conte de fées dans sa plus haute acception, chez Perrault comme chez Mendès, recourt volontiers à la satire, qui en retour garantit sa véridicité ou, si l'on veut, son «mentir vrai».

Mais avec l'excès et ces figures que sont l'accumulation et l'hyperbole, on touche à des traits qui caractérisent non seulement le merveilleux en luimême, mais l'usage qu'en font nos deux auteurs. Ainsi, dans la fin bien connue de "Riquet à la houppe», c'est encore la satire qui provoque une exagération comique. On se souvient de la métamorphose miraculeuse de Riquet sous l'effet de l'amour de la princesse, version que le conteur s'empresse de contester au profit d'une interprétation allégorique (reprise d'ailleurs dans la première moralité):

8. Voir à cet égard le commentaire par J. Stora-Sandor du concept d'ironie chez Kierkegaard, L'Humour juif dans la littérature, Paris, Presses universitaires de France, 1984, p. I8-19.

9. J'entends le terme au sens que $\mathrm{M}$. Kundera, à la suite de H. Broch, lui a donné dans L'Insoutenable Légèreté de l'être (1984) et L'Art du roman: «le besoin de se regarder dans le miroir embellissant du mensonge et de s'y reconnaître avec une satisfaction émue» (Paris, Gallimard [NRF], I986, p. I64). 
Quelques-uns assurent que ce ne furent point les charmes de la Fée qui opérèrent, mais que l'amour seul fit cette Métamorphose. Ils disent que la princesse ayant fait cette réflexion sur la persévérance de son amant, sur sa discrétion, sur toutes les bonnes qualités de son âme et de son esprit, ne vit plus la difformité de son corps, ni la laideur de son visage, que sa bosse ne lui sembla plus que le bon air d'un homme qui fait le gros dos, et qu'au lieu que jusqu'alors elle l'avait vu boiter effroyablement, elle ne lui trouva plus qu'un certain air penché qui la charmait; ils disent encore que ses yeux, qui étaient louches, ne lui en parurent que plus brillants, que leur dérèglement passa dans son esprit pour la marque d'un violent excès d'amour, et qu'enfin son gros nez rouge eut pour elle quelque chose de Martial et d'Héroïque? (p. 289)

Cela fait beaucoup (et l'étrange point d'interrogation final peut figurer le doute ironiquement intégré à la narration elle-même). Car à tout prendre, l'explication merveilleuse était finalement beaucoup moins invraisemblable que celle par les causes rationnelles. Que reste-t-il ? Rien, sinon à conclure, en donnant au conte son finale obligé. "Quoi qu'il en soit» (ibid.) - puisque telle est la formule plus rhétorique que magique qui précipite la conclusion il a fallu excessivement de pouvoir chez la fée ou excessivement d'amour chez la princesse. On ne peut s'empêcher de songer - et Perrault s'en souvient sans doute - au Misanthrope et à la tirade au cours de laquelle Éliante raille avec brio l'aveuglement des "amants " ${ }^{\mathrm{IO}}$. Mais alors que, chez Molière, à chaque "objet aimé» correspondait un seul défaut, Perrault s'amuse à les accumuler tous chez Riquet, au point que le premier excès du renversement - un défaut se transformant en élément de séduction - se double d'un second, par lequel tous ces défauts - ou toutes ces "perfections" - se trouvent concentrés sur la personne du Prince Riquet. C'est là un des moments les plus franchement comiques des Contes, par la vision double et contradictoire qu'il instaure d'un prince qui ressemble à tout sauf à un prince tel que les contes de fées ont coutume de le représenter, y compris chez Perrault lui-même. Le comique satirique se renforce donc d'une parodie probable, mais également d'un jeu parodique plus large avec les règles et les attentes d'un genre qu'il prend à contre-pied. C'est le merveilleux lui-même, en somme, qui est systématiquement mis en question.

\section{Le merveilleux parodique}

On a vu que l'excès et l'accumulation étaient tout à la fois les signes du merveilleux et de sa mise à distance parodique. Perrault en joue volontiers lorsqu'il oppose le dénuement très réaliste du jeune maître du Chat botté à l'étendue

Io. Tirade fameuse de l'acte II, sc. 4, v. 7II-730, elle-même démarquée du De rerum natura de Lucrèce que Perrault devait aussi connaittre, ce qui ajoute une strate supplémentaire au palimpseste parodique. 
des richesses de l'ogre, ou lorsqu'il narre la visite émerveillée des amies de la jeune mariée dans «La Barbe Bleue»:

Les voilà aussitôt à parcourir les chambres, les cabinets, les garde-robes, toutes plus belles et plus riches les unes que les autres. Elles montèrent ensuite aux garde-meubles, où elles ne pouvaient assez admirer le nombre et la beauté des tapisseries, des lits, des sophas, des cabinets, des tables et des miroirs, où l'on se voyait des pieds jusqu'à la tête, et dont les bordures, les unes de glace, les autres d'argent et de vermeil doré, étaient les plus belles et les plus magnifiques qu'on eût jamais vues. (p. 262-263)

La chute est récurrente dans les Contes, et pour tout dire canonique. Ce sont pourtant la sobriété et l'économie qui caractérisent plutôt la manière de Perrault, qui, en général, cultive moins la description et l'accumulation que les caractérisations très fortement codées - «les unes de glace, les autres d'argent et de vermeil doré» - ou tout simplement évaluatives: «les plus belles et les plus magnifiques qu'on eût jamais vues». Et bien souvent, les indices du merveilleux dispensent de le décrire: "Ils entrèrent dans une grande salle où ils trouvèrent une magnifique collation" («Le Chat botté», p. 27I). Par là, Perrault laisse le champ libre à Mendès, qui se fait un plaisir de combler les lacunes de son devancier, et se livre à un exercice de style dans la surenchère. En proposant une variation explicite sur son modèle, Mendès donne ainsi la preuve de sa virtuosité de conteur. À preuve l'incipit de «La Belle au bois rêvant»:

[...] il faut bien reconnaitre qu'il est arrivé aux conteurs les plus consciencieux, les mieux informés - Mme d'Aulnoy, le bon Perrault lui-même, - de ne pas relater les choses exactement de la façon qu'elles s'étaient passées dans le pays de la féerie. Ainsi, l'aînée des sœurs de Cendrillon ne portait pas au bal du prince, comme on l'a cru jusqu' ici, un habit de velours rouge avec une garniture d'Angleterre; elle avait une robe d'écarlate, brodée d'argent et passementée d'orfroi. Parmi les monarques de tous les pays, priés aux noces de Peau d'Âne, les uns, en effet, vinrent en chaise à porteurs, d'autres en cabriolet; les plus éloignés montés sur des éléphants, sur des tigres, sur des aigles; mais on a omis de nous faire savoir que le roi de Mataquin fit son entrée dans la cour du palais, assis entre les ailes d'une tarasque qui jetait par les naseaux des flammes de pierreries. (p. 52-53)

On le voit, Mendès corrige Perrault, et ce pour plusieurs raisons. La première est évidemment de préparer et de légitimer le retournement parodique qu'il va faire subir à la version officielle du conte. Mais il n'en tire pas moins au passage quelques autres bénéfices secondaires, et d'abord celui de donner une image paterne de Perrault - «le bon Perrault»! - au prix d'un adoucissement ou d'un affadissement tout à fait abusif de l'image du conteur que deux siècles de réception ont favorisés. Du coup, Mendès peut mettre en valeur son traitement du merveilleux - et notamment l'humour dont il fait preuve à cet 
égard - comme un trait profondément original. Or nous savons que, sur ce plan déjà, Mendès est à bonne école avec Perrault. Mais il n'en est pas moins vrai que son prétendu scrupule d'exactitude et de réalisme ne manque pas de saveur sur de telles matières. C'est pourquoi on ne saurait s'étonner que la complicité parodique s'instaure bien davantage avec Perrault qu'avec Mme d'Aulnoy! Dans le défi lancé à l'illustre prédécesseur sur un ton badin, il y a aussi une forme de toupet qui ne manque pas de drôlerie, parce que sa légèreté suffit à donner la mesure de son iconoclasme. Mais là où Mendès se montre inégalable, c'est dans l'abondance et le luxe de la description (qui d'ailleurs n'intéressaient manifestement pas Perrault). Il y montre néanmoins une verve et une virtuosité réjouissantes, comme dans l'évocation des noces de Peau d'Âne qu'il amplifie en en respectant le style. Dans un autre conte, Mendès propose une variation sur le même thème:

[Les fées] ne manquèrent pas de venir, celles-ci, dans des litières de drap d'or, aux franges de pierreries, que portaient des Africains nus, celles-là dans des chars de cristal, attelés de quatre unicornes: il y en eut qui trouvèrent plus commode d'entrer par la fenêtre ou par la cheminée, sous forme d'oiseaux de paradis ou de martinets aux ailes bleues; mais, dès qu'elles frôlaient le parquet de la salle, elles devenaient de belles dames habillées de satin. ("Les Accordailles», p. II3-II4)

Les matériaux précieux et l'exotisme participent tout autant du merveilleux que les prouesses surnaturelles. Il y a là aussi une forme de "débauche" complaisante qui nous suggère que, chez Mendès, l'érotisme est presque toujours l'horizon du merveilleux, qu'il s'agisse des "Africains nus» ou des oiseaux bleus qui se métamorphosent - en quelque sorte avantageusement en «belles dames habillées de satin». Cet érotisme omniprésent, nous le retrouvons jusque dans le souper offert au prince "convié des fées", - événement qui en lui-même ne saurait se produire chez Perrault, ni par l'imaginaire qu'il suppose, ni par le raffinement quelque peu mignard auquel il donne lieu:

[...] ce qui le charma surtout ce fut le dessert, la trace d'un baiser d'abeille sur un pétale de rose. («Le Mauvais Convive», p. 124)

Reste néanmoins l'humour, dans l'évocation poétique de ce nectar d'amour quintessencié, nourriture trop subtile pour un être humain, fût-il poète. Aussi le prince meurt-il «d'inanition», car telle est la morale du conte:

C'est qu'il était un de ces pauvres êtres - tels sont les poètes ici-bas - trop purs, et pas assez, trop divins pour partager les festins des hommes, trop humains pour souper chez les fées. (p. I25-I26)

Par ce nouveau biais, Mendès conteste le merveilleux qu'on pourrait aussi bien nommer idéalisme poétique. Quant au merveilleux de la féerie, nul n’est 
plus assez naïf pour y croire - ce serait un des leitmotive des contes. Étonnante morale de conte de fées, si l'on y songe, que celle qui fait du merveilleux luimême - ou de la poésie - une illusion aussi belle que pernicieuse. On voit bien là ce qui rapproche et sépare tout à la fois Perrault et Mendès: si tous deux manifestent clairement leur distance par rapport à la féerie, le premier sait qu'il appartient à un monde désenchanté, alors que le second le voit en outre touché par la mélancolie. Du premier au second, il semble que la perte du merveilleux soit devenue une question lancinante dans une société largement déchristianisée qui n'a plus guère d'exutoires pour ses aspirations et ses rêves. C'est pourquoi le rire des deux conteurs résonne dans des registres distincts, lors même qu'il porte sur une narration qu'il leur incombe à tous deux de réenchanter malgré tout.

\section{La désinvolture narrative, ou le jeu à l'égard des codes}

Nos deux conteurs affichent donc une distance amusée qui, au-delà de la matière elle-même, touche le protocole en vertu duquel tout narrateur doit ajouter foi à l'histoire qu'il raconte. Rien de tel ici, par exemple lorsque la princesse de «La Belle au bois dormant» se blesse avec le fuseau:

Elle n'eut pas plutôt pris le fuseau, que comme elle était fort vive, un peu étourdie, et que d'ailleurs l'Arrêt des fées l'ordonnait ainsi, elle s'en perça la main, et tomba évanouie. (p. 249-250)

Curieusement, la troisième explication, en délaissant le caractère de la princesse pour une fatalité extérieure, mine le sérieux de l'ensemble, puisque les deux premières deviennent rétrospectivement inutiles. Bien plus, le glissement est insensible de l' "Arrêt des fées " à la nécessité interne du conte, dont Perrault prend manifestement plaisir à souligner le fonctionnement. S'agit-il des personnages, Perrault les campe à l'occasion en modèles purement littéraires, non sans un clin d'œil à l'adresse de son lecteur. Ainsi du prince en marche vers le château de la belle au bois dormant: «Il ne laissa pas de continuer son chemin : un Prince jeune et amoureux est toujours vaillant» (p. 25I). Où Perrault serait-il allé chercher cette vérité, sinon dans les contes euxmêmes? Or c'est là un des traits du génie de Perrault: intéresser son lecteur à une histoire, tout en jouant avec des stéréotypes qu'il désigne comme tels. Mendès lui emboîte le pas, condensant en une phrase tous les signes attendus de l'élection d'un jeune homme inconnu:

Or, il arriva une fois qu'un jeune pêcheur, fort bien fait de sa personne, et très agréable à voir malgré ses haillons de bure, entra dans la cour du palais, et dit, avec un air d'assurance, qu'il voulait parler au roi. ("La Bonne Récompense», p. 139) 
On sait qu'être "bien fait de sa personne» - entre autres mérites - tient tout à fait lieu de rang princier dans les contes, et permet à plus d'un d'épouser la fille du roi. "Le Chat botté» nous l'avait déjà appris - ou presque -, lorsque le prétendu marquis de Carabas était présenté au roi :

Le Roi lui fit mille caresses, et comme les beaux habits qu'on venait de lui donner relevaient sa bonne mine (car il était beau, et bien fait de sa personne), la fille du Roi le trouva fort à son gré et le comte de Carabas ne lui eut pas jeté deux ou trois regards fort respectueux, et un peu tendres, qu'elle en devint amoureuse à la folie. (p. 269)

Certes, en cet âge aristocratique, la bonne mine ne suffit pas tout à fait comme chez Mendès, et il faut pour la relever quelques «beaux habits» et une imposture, car dans la féerie classique, il est tout de même plus difficile à un roturier d'épouser une princesse qu'à une bergère d'épouser un prince. Mais ce qui est surtout amusant, c'est l'ajout désinvolte et entre parenthèses d'un trait aussi essentiel qui n'est précisé qu'au moment où le besoin s'en fait sentir (ce qui est une manière d'en exhiber l'artifice). À plusieurs reprises, Perrault joue de ces omissions prétendues et de ces précisions après coup qui miment peut-être les aléas de la narration orale, mais montrent surtout la liberté de l'auteur à l'égard des moindres conventions narratives: c'est là que réside sa fraîcheur, qui est un effet de l'art. La phrase qui conclut le premier alinéa de «Riquet à la houppe» nous en fournit un des plus beaux exemples:

J'oubliais de dire qu'il vint au monde avec une houppe de cheveux sur la tête, ce qui fit qu’on le nomma Riquet à la houppe, car Riquet était le nom de la famille. (p. 285)

Il était grand temps en effet de signaler cette particularité qui fournit le titre du conte, mais Perrault donne à tout cela le naturel et le caractère imparable d'une logique enfantine, et il met ainsi de son côté les rieurs - qui seront les grandes personnes. Car «le prince Riquet», c'est déjà en soi un oxymore burlesque. La désinvolture de Mendès emprunte quant à elle des voies légèrement différentes: non pas la précision après coup, mais la précision oiseuse, comme dans «La Belle du monde»:

Une fois que, sur la pelouse, avec ses demoiselles d'honneur, elle jouait à la berlurette - c'était un jeu fort à la mode, en ce temps à la cour, - elle entendit deux pages [...]. (p. 36)

Et plus loin:

Un matin que, dans une galerie, parmi ses demoiselles d'honneur, elle jouait au baguenaudier - c'était un jeu qui, en ce temps-là, à la cour, n'était pas moins à la mode que la berlurette, - elle entendit deux officiers du palais [...]. (p. 40) 
On perçoit là tout d'abord un écho de la formule démystificatrice de Perrault au début de "La Belle au bois dormant " : "comme c'était la coutume des Fées en ce temps-là» (p. 247). Mais Mendès excelle aussi dans le jeu sur le merveilleux verbal par le cachet qu'il sait donner à des mots inconnus et suggestifs: on y perçoit malgré tout «berlue» et «baguenaude(r)», ce qui, dans les deux cas, revient à dire l'illusion ou la bagatelle d'un rien (ou quelque chose d'aussi frivole qu'un conte?), et à feindre d'expliquer l'obscur par le plus obscur encore. Ce faisant, Mendès joue aussi de l'amorce narrative canonique des contes par la mise en relief des temps verbaux, le récit proprement dit étant comme à plaisir différé. Le redoublement du procédé, son signalement et le jeu subtil de variation jusque dans la distribution stylistique des termes des deux propositions entre tirets, tout montre ici un art de conter consommé et concerté, et qui semble s'enchanter de ce talent dont il n'est d'ailleurs pas dupe. Ce n'est pas un hasard si, pour Mendès, le merveilleux n'est jamais au fond que la poésie elle-même, en somme le pouvoir du langage.

Il est néanmoins chez Perrault une place stratégique où éclatent le plaisir et la gratuité de l'artifice: ce sont les moralités. Le conteur, comme on sait, n'en est pas avare, et il en fournit souvent plusieurs. Mais paradoxalement et pour cette raison même, celles-ci vont plutôt à l'encontre du caractère exemplaire du genre ${ }^{\text {II }}$. On pense volontiers à un divertissement de salon où chacun ferait assaut d'esprit et dont la consigne pourrait se formuler en ces termes: soit le conte que vous venez d'entendre: à votre tour proposez une moralité (en vers), et si possible spirituelle. Ainsi, dans «Riquet à la houppe», après une première moralité qui accrédite l'interprétation allégorique par le pouvoir magique attribué à l'amour, Perrault en propose une seconde, beaucoup plus subtile et précieuse:

\author{
Dans un objet où la Nature \\ Aura mis de beaux traits, et la vive peinture \\ D'un teint où jamais l'Art ne saurait arriver \\ Tous ces dons pourront moins pour rendre un cour sensible, \\ Qu'un seul agrément invisible \\ Que l'Amour y fera trouver. (p. 289)
}

À l'évidence, cette moralité ne peut se déduire strictement du conte, puisqu'elle en conteste la lettre sur un point essentiel - tout le conte reposant en effet sur la laideur initiale de Riquet. Mais c'est pour l'enrichir aussitôt d'une valeur ajoutée: la mise en évidence d'un charme "invisible» qui échappe à la peinture. Le conte sert donc ici de pré-texte et de tremplin à une moralité qui vise plus haut - c'est son caractère précieux -, tout en se jouant

II. Voir sur la notion de «récit exemplaire» S. Suleiman, Le Roman à thèse, Paris, Presses universitaires de France (Écriture), 1983, p. 35-78. 
de l'argument du conte. Mais les moralités de Perrault peuvent aussi varier selon les circonstances des histoires, et celle du "Chat botté» par exemple, qui met en avant «l'habit, la mine et la jeunesse / Pour inspirer de la tendresse» (p. 272), contredit largement celle de "Cendrillon", selon laquelle tous les talents du Ciel ne seraient rien sans l'appui «Ou des parrains ou des marraines» (p. 283) ${ }^{\mathrm{I} 2}$. On voit assez que les moralités admettent de la souplesse et ce qu'il faut bien appeler $d u j e u$.

Mais il arrive aussi, comme pour "La Belle au bois dormant», que la moralité en rabatte singulièrement par rapport au propos du conte, et qu'elle s'en tienne à un trait de satire burlesque volontairement réducteur :

\section{Attendre quelque temps pour avoir un Époux \\ Riche, bien fait, galant et doux, \\ La chose est assez naturelle, \\ Mais l'attendre cent ans, et toujours en dormant \\ On ne trouve plus femelle \\ Qui ne dormît si tranquillement. (p. 257)}

Assurément, une telle moralité est à mettre au compte d'un moraliste qui se soucie bien peu d'édifier. Et deux cents ans plus tard, Mendès se ferait scrupule de dire de telles choses aussi franchement. L'auteur des Oiseaux bleus se garde bien d'ailleurs d'assortir ses contes de moralités séparées. Le temps en est passé, et la morale est devenue en quelque sorte inhérente à la fable, qu'elle jalonne de réflexions: "[...] chez les fées, c'est comme chez les hommes: on est bon quand on est content, mécontent quand on est triste». ("Isolin-Isoline», p. 68) ${ }^{\text {r3 }}$; ou encore: "Il suffit souvent d'une caresse ou d'un souffle de femme pour qu'une aile tombe» («L'Ange boiteux», p. 203). La moralité trouve également place dans la conclusion, où elle est quelquefois explicitement présentée comme telle. La «sagesse» du conteur s'y teinte alors d'humour comme dans le conte des "Trois Semeurs». Trois jeunes compagnons y voient leur vœu exaucé par une fée: le premier, celui de puissance; le deuxième, celui de richesse; et le troisième - le poète -, celui plus modeste de chanter l'épithalame qu'il a composé la veille pour «l'hymen de deux fauvettes». Et il y gagne aussitôt de voir

[...] une belle jeune femme à demi nue - si belle qu'aucun rêve d'amour l'eût souhaitée plus parfaite, - sorti[r] de la terre entrouverte, et mettant ses deux bras,

I2. Certes, elle ne la contredit pas sur l'esprit, puisqu'en l'espèce un chat vaut bien une fée marraine, mais encore faut-il retourner aux contes pour s'en persuader, alors que le mouvement de la moralité est plutôt de s'en abstraire.

I3. C'est fournir là en outre un commentaire sur Perrault et une variation sur l'incipit de «La Belle au bois dormant", car il s'agit d'opposer deux fées, l'une invitée, l'autre non, au baptême de la fille du roi. 
lianes pour l'enlacement et lys pour la blancheur, au cou de l'enfant ravi: «Oh! comme tu chantes bien! Je t'aime! lui dit-elle». (p. I83)

Dans les lignes qui suivent, Mendès a tôt fait d'expédier la fin du conte: de soudains revers de fortune brisent les destinées des deux premiers compagnons en moins de temps qu'il n'en avait fallu à une fée pour les relever. Mais le conteur épargne le dernier compagnon, et le récit se résout sur l'accord parfait d'une félicité réservée au plus fortuné des mortels:

Seul, Aloys ne cessa point d'être heureux, baisé du soir au matin, et du matin au soir, par la belle jeune femme dont les bras souples comme des lianes étaient blancs comme des lys; et elle lui fut fidèle, toujours, toujours, parce qu'il avait chanté dans le sillon féerique une chanson bien rimée. (p. I84)

Tout finit donc par une chanson. Conserver le pouvoir de la féerie quand même, et jouir en toute lucidité du pouvoir de séduction d'un beau mensonge dont on se moque dans le même temps - car qui peut croire, même chez les fées qui ne sont plus nées de la dernière pluie, pareille histoire à dormir debout? -, telle est sans doute la posture du conteur moderne, qui écartèle les postulations de la naïveté et de l'incrédulité. Celles-ci étaient déjà présentes chez Perrault, mais sans être nouées par une dialectique aussi serrée - et j'ajouterais presque, aussi porteuse d'implications philosophiques. Car paradoxalement, la croyance de celui qui ne croit plus, et qui veut continuer à croire précisément parce qu'il a cessé de croire ${ }^{\mathrm{I} 4}$, trace exactement le cercle métaphysique dans lequel elle s'inscrit. C'est pourquoi, une fois encore, l'humour de Mendès repose sur un fond plus grave que celui de Perrault. Mais ne forçons pas trop le trait. Voilà donc une fausse moralité, ou une moralité pour rire, que dénoncent à la fois la contre-vérité topique sur la fidélité des femmes et son emphase lyrique surjouée. C'est qu'assurément Mendès ne compte pas pour rien le plaisir du rire, ni celui du jeu, ni enfin celui d'une poésie qui se met en scène et se contemple elle-même dans le miroir du conte. Surtout, comme dans «Les Mots perdus» ("mots» d'amour, bien sûr, et "perdus» par le sortilège d'une méchante fée), la poésie est proprement douée du pouvoir d'enchanter un monde voué à la prose. Ainsi finit le récit:

Car c'est à cause des vers que les baisers sont doux, et les amoureux ne disent rien que les poètes n'aient chanté. (p. I5I)

On comprend dès lors la signification allégorique du conte: la poésie est elle-même cette fée sans le secours de laquelle rien de l'existence humaine ne vaut vraiment d'être vécu. Au commencement était donc non pas la vie, mais

I4. Celle-ci a été analysée sur le plan psychanalytique par O. Mannoni sous la forme du «Je sais bien, mais quand même» dans Clefs pour l'imaginaire ou l'Autre Scène, [1969], Paris, Seuil (Points), 1985, p. 9-33. 
le verbe, - entendons la poésie, ou la littérature, ou l'imaginaire: renversement décadent si l'on veut, mais qui n'en est pas moins un acte de foi - le seul! - qu'il convient ici de prendre au sérieux. Et de là à dire que la poésie - ou les rêves - valent mieux que la vie, il n'y a qu'un pas que l'auteur de «La Belle au bois rêvant" va s'empresser de franchir. Car Mendès fait bifurquer le conte de Perrault, qu'il a jusque-là suivi à la lettre ${ }^{\mathrm{I}}$, à partir du réveil de la princesse. Celle-ci, loin de marquer au prince le plaisir de sa venue et de répondre avec empressement à son amour, lui demande d'énumérer les plaisirs qui l'attendent «dans la clarté et dans la vie» qu'il lui promet (p. 56). Mais ni la puissance, ni le fait d'être courtisée, ni les toilettes splendides - dont les « robes couleur de lune et de soleil» (p. 57) sont directement empruntées à Perrault-, ni les délices de la table, ni même l'amour d'un prince, rien ne peut la convaincre. Il faut donc écouter sa réponse:

Je dors depuis un siècle, c'est vrai, mais depuis un siècle, je rêve. Je suis reine aussi, dans mes songes, et de quel divin royaume! Mon palais a des murs de lumière; j'ai pour courtisans des anges qui me célèbrent en des musiques d'une infinie douceur, je marche sur des jonchées d'étoiles. Si vous saviez de quelles robes je m'habille, et les fruits sans pareils que l'on met sur la table, et les vins de miel où je trempe mes lèvres! Pour ce qui est de l'amour, croyez bien qu'il ne me fait pas défaut; car je suis adorée d'un époux plus beau que tous les princes du monde et fidèle depuis cent ans. Tout bien considéré, monseigneur, je crois que je ne gagnerais rien à sortir de mon enchantement; je vous prie de me laisser dormir. (p. 59)

Quelle est donc la morale de cette chute peu morale? Certes, nous reconnaissons en la princesse une petite personne très fin de siècle, à qui manquent les vertus de bonté et d'honnêteté célébrées par Perrault. Ou, pour parler comme Alphonse Allais, voilà décidément "une petite femme bien moderne»! Ce serait la part de satire greffée sur la parodie. Mais il n'empêche que le conte n'en peint pas moins - et avec quelles couleurs! - l'irrésistible séduction du rêve qui, par la syllepse du mot «enchantement", transfere la magie du pouvoir de la fée à celui de la rêveuse comme à celui du conteur, magicien à son tour pour avoir su évoquer les prestiges du rêve. C'est là le seul cas de parodie stricte et complète d'un conte de Perrault par Mendès. S'il s'agit d'un Perrault "pris à rebours et contesté dans ses principes " (Jean de Palacio, "préface», p. 9), j’y verrai aussi volontiers, au-delà du jeu de perversion, une suprême affirmation du pouvoir de la poésie - cette fois présentée sous la forme du rêve. Or Mendès sait bien que Perrault ne la méconnaît pas, puisque c'est principalement chez lui qu'il la puise. Sur le plan narratif, c'est peut-être

I5. " [...] une princesse qui paraissait avoir quinze ou seize ans, et dont l'éclat resplendissant avait quelque chose de lumineux et de divin» (op. cit., respectivement p. 25I et 55). 
moins la remise en cause des "principes» de Perrault qui produit le renversement parodique que leur systématisation et leur usage exclusif. Car le parti pris exclusif du merveilleux est non seulement la négation du réel, mais aussi de la vie, et Mendès ne laisse pas de jouer de cette fascinante ambiguïté.

Sur les points abordés dans ce parcours - la satire, la parodie du merveilleux, et celle qui touche les codes de la narration - on est loin d'avoir épuisé la poétique du conte de fées propre à Perrault, pas plus d'ailleurs que celle de Mendès. On voudrait néanmoins avoir mis en évidence une intersection, ou mieux, un lieu de rencontre. Ce que Perrault et Mendès ont en commun tient en effet au déplacement du merveilleux - auquel ni l'un ni l'autre ne croient plus -, puisqu'il migre du contenu vers une narration consciente de ses pouvoirs et qui, dans les deux cas, confere au conte la magie du verbe, ou poésie. Poésie, sans doute, mais chez l'un comme chez l'autre poésie rieuse - où je verrais pour ma part une autre complicité profonde. Cela n'empêche nullement que la poésie selon Mendès soit beaucoup plus réflexive, autoréférentielle et finalement poéticienne. C'est pourquoi son merveilleux - même nié - finit toujours par s'identifier à la poésie comme ultime valeur: on aura reconnu là le moment Mallarmé.

Mais on en vient également à se demander si la fin du XIXe siècle a tant aimé les contes de fées - et en particulier ceux de Perrault -, non pas tant parce qu'ils auraient présenté une matière "brute» qu'elle pouvait à loisir recycler et parodier - ce serait le mythe -, mais bien plutôt parce qu'ils lui proposaient déjà des ouvres d'une forme raffinée et savamment élaborée, dont les très puissants enjeux imaginaires semblaient aussi préfigurer sa propre esthétique. Certes, entre-temps, le ton a changé et le rire s'est assombri ou teinté de mélancolie. S'il reste néanmoins une verve toute neuve et parfois une allégresse native dans le plaisir de conter, c'est chez Catulle Mendès qu'on la trouve, et on ne renoncera pas à y lire l'hommage d'une littérature de grande santé - malgré tout - au génie de Charles Perrault. 